\title{
WAVE-DRIVEN ROTATION IN SUPERSONICALLY ROTATING MIRRORS
}

\author{
ABRAHAM J. FETTERMAN* and NATHANIEL J. FISCH \\ Princeton University, Department of Astrophysical Sciences, Princeton, New Jersey 08540
}

Received November 24, 2009

Accepted for Publication January 25, 2010

Supersonic rotation in mirrors may be produced by radio-frequency waves. The waves produce coupled diffusion in ion kinetic and potential energy. A population inversion along the diffusion path then produces rotation. Waves may be designed to exploit a natural kinetic energy source or may provide the rotation en- ergy on their own. Centrifugal traps for fusion and isotope separation may benefit from this wave-driven rotation.

KEYWORDS: magnetic mirrors, rotation, radio-frequency waves

\section{INTRODUCTION}

Magnetic mirror traps with supersonic rotation have many possible uses, including fusion energy and isotope separation. ${ }^{1-7}$ For fusion applications, the rotation produces enhanced axial confinement and magnetohydrodynamic (MHD) stability. ${ }^{8-11}$ Plasma centrifuges for isotope separation have the advantage of larger rotation speeds than gas centrifuges, leading to higher separation factors. ${ }^{12,13}$ In both of these applications, a significant technical hurdle has been the presence of electrodes or end insulators, at the surface of which the rotation may be limited to the Alfven critical ionization velocity (CIV) (Refs. 1, 14, and 15). The electrodes have been necessary to create a radial electric field, which causes the plasma to rotate by the $E \times B$ drift. We have shown that instead of driving current through electrodes, the rotation could be produced volumetrically by radio-frequency waves near the ion cyclotron frequency. ${ }^{16,17}$

To appreciate the usefulness of these waves, we may consider the issues that have been critical to electrode design. The limitation imposed by the Alfven CIV has been the most difficult to overcome, appearing in different applications across many orders of magnitude in pressure and temperature. ${ }^{15}$ The critical velocity is $v_{c}=\sqrt{2 e \phi_{i} / m_{n}}$, where $\phi_{i}$ is the ionization potential of neutrals and $m_{n}$ is their mass. As the power supplied to the plasma is increased to raise the rotation velocity

\footnotetext{
*E-mail: afetter@pppl.gov
}

above this threshold, the power only produces electron heating and increased ionization. The mechanism responsible for instability has been the subject of some debate as a result of conflicting data from space-based experiments. ${ }^{18}$

Several experiments have succeeded in surpassing the Alfven CIV at the electrode surface. The only fusionrelevant scheme to do so has been the PSP-2 experiment in Novosibirsk. ${ }^{19}$ Velocities exceeding the Alfven CIV by a factor of 40 were achieved by careful electrode and liner design, strong vacuum conditions, and extensive conditioning. The Alfven CIV has also been exceeded in plasma centrifuges of the vacuum-arc type. ${ }^{6,20}$ These devices feature strong vacuum conditions and a highly ionized plasma. However, they may be limited in separative power by the small column radius and single-direction flow. $^{21-23}$

Besides the limitation due to the Alfven CIV, the use of electrodes to drive rotation may restrict the achievable rotation through other considerations. ${ }^{24}$ If the electrodes are placed in the mirror throat, there must be sufficient plasma there to provide parallel conductivity to the bulk plasma. If the rotation profile is instead determined by the perpendicular conductivity (for example, by using an electrode along the center axis), ionization and MHD instabilities may develop in the plasma.

One can see that it would be desirable if instead of relying on current driven by electrodes, the rotation could be maintained by current drive within the bulk plasma. Rotation speeds small compared to the ion temperature 
have been observed in tokamaks, and one possible mechanism for this is the presence of waves near the ion cyclotron frequency. ${ }^{25}$ The rotation may be produced by absorption of wave momentum or by neoclassical ion viscosity. ${ }^{26-28}$ We will see that rotation may be produced in a similar manner in centrifugal mirror traps and that with appropriate waves this can be done efficiently.

The primary source of momentum drag in most centrifugal mirrors is the formation of new ions. ${ }^{3}$ When a neutral is ionized, the heavy ion moves one gyroradius toward the negative electrode while the electron is nearly stationary, producing a charge separation. Processes of thermalization and transport are ambipolar, so that this charge separation is maintained despite collisions. ${ }^{29}$ The energy lost due to the charge separation is provided by the external power source once both the ion and electron are lost axially.

With wave-induced ion diffusion, the ions interact with the wave and travel radially within the plasma, but electrons do not. If ions that move toward the positive electrode are allowed to exit but ions that move toward the negative electrode are not, the average charge separation is decreased. This means that less energy must be supplied by the electrodes to maintain the plasma. If the average radial distance the ion travels before exiting is at least the initial gyroradius, the electrodes may be eliminated entirely. Resonant particles can only absorb energy and momentum in a ratio proportional to their velocity, which is $\omega / k$. This momentum is ultimately balanced by the reactive force of the wave on the antenna. This will be the foundation for producing plasma rotation with waves. ${ }^{16}$

First, this paper will discuss the branching ratio, a new term to describe wave-particle interactions in rotating plasmas. Then, the method for using waves to produce rotation will be described. Finally, we will show how waves can be used to drive rotation in fusion plasmas and plasma centrifuges.

\section{BRANCHING RATIO}

When a particle interacts with a wave, it must absorb or emit wave momentum and energy in the same proportions that they exist in the wave. For example, the particle cannot reduce the wave energy but increase the wave momentum. This leads to a strong constraint on the change in the constants of motion of the particle. If a particle interacts repeatedly with the same wave at random phase, its motion can be described by a diffusion path in phase-space.

This path describes constrained diffusion not only in velocity but also in position. To see this, consider a slab geometry with a uniform magnetic field in the $z$-direction and a uniform electric field in the $y$-direction. Shift to the frame moving with $v_{E}=E_{y} / B_{z}$, denoting terms in this frame with a tilde. The particle is moving in circular orbits in this frame, as there is no electric field.
A wave with phase velocity $\tilde{v}_{p h}=k_{x} / \widetilde{\omega}$ interacts with a particle moving with $\tilde{v}_{x}=\tilde{v}_{p h}$. If the velocity is changed by $\Delta v_{x}$, this will lead to a change in the gyrocenter position $\Delta y_{g c}=-\Delta v_{x} / \Omega_{i}$, where $\Omega_{i}$ is the gyrofrequency. The change in velocity leads to the change in energy $\Delta \widetilde{W}=m_{i} \tilde{v}_{x} \Delta v_{x}$, so that using the resonance condition we find $\Delta y_{g c}=-\Delta \widetilde{W} / \tilde{v}_{p h} \Omega_{i} m_{i}$. From this relationship, we see that if $\tilde{v}_{p h}>0$, the particle must move down if it gains energy and must move up if it loses energy.

In the moving frame, the interaction appears to be only between the particle and the wave, since there is no electric field. ${ }^{30}$ However, if we consider the interaction in the lab frame, the $E_{y}$ field can be seen to play a significant role. Since the particle gyrocenter has moved by $\Delta y_{g c}$, there is a change in the potential energy $q \Delta \Phi=$ $-q E_{y} \Delta y_{g c}$. Substituting our previous result, we find $q \Delta \Phi=v_{E} \Delta \widetilde{W} / \tilde{v}_{p h}$. This relationship is the basis for the branching ratio $f_{E}=-v_{E} / \tilde{v}_{p h}$.

The branching ratio is the increase in the potential energy divided by the total change in the particle energy. ${ }^{16}$ For $\left|f_{E}\right| \ll 1$, energy is primarily transferred between the wave and the particle through kinetic energy, as in the stationary mirror case. ${ }^{31,32}$ However, if $\left|f_{E}\right| \geq 1$, the potential energy change is at least as large as the kinetic energy change.

To apply the branching ratio to a rotating plasma, we must introduce the centrifugal force and Coriolis effect. The first leads to an additional drift, and the second leads to an effective change in the cyclotron frequency. ${ }^{33}$ We define the ratio $\chi=v_{E} / r \Omega_{i}$, so that the rotation frequency $\Omega \approx \Omega_{i}\left(\chi-\chi^{2}\right)$ and the cyclotron frequency $\widetilde{\Omega}_{i} \approx \Omega_{i}(1+2 \chi)$ for small $\chi$. Defining $n_{\theta}=k_{\theta} / r$, we find the branching ratio $^{17}$

$$
f_{E} \approx \frac{-n_{\theta} \chi}{(1+4 \chi) \widetilde{\omega} / \widetilde{\Omega}_{i}+n_{\theta} \chi^{2}} .
$$

Note that Eq. (1) is a minor correction of Eq. (7) in the reference. ${ }^{17}$ One can see the full solution in terms of $\chi$ and $n_{\theta} \widetilde{\Omega}_{i} / \widetilde{\omega}$ in Fig. 1 (for perpendicular waves, the $y$-axis is just $\left.n_{\theta}\right)$. We note that for $\chi<-\frac{1}{4}$ particles are untrapped, as in the band gap ion mass filter. ${ }^{7}$ We have divided the space into three regions based on the type of interaction. Let us consider these regions one at a time.

\section{Region I}

The simplest case, $f_{E} \approx 0$, is familiar as particle heating in a nearly stationary plasma. At $f_{E}=0$ exactly, there is no change in the potential energy. For $0<f_{E}<$ 0.5 (the darker part of Region I), there is a small increase in potential energy as the kinetic energy decreases, while for $-1<f_{E}<0$ the opposite is true. The radial motion of the particle may be seen as the drift due to an average accelerating force, or as absorption of wave momentum.

\section{Region II}

In this region around $f_{E}=1$, kinetic and potential energy are interchanged and wave energy is nearly 


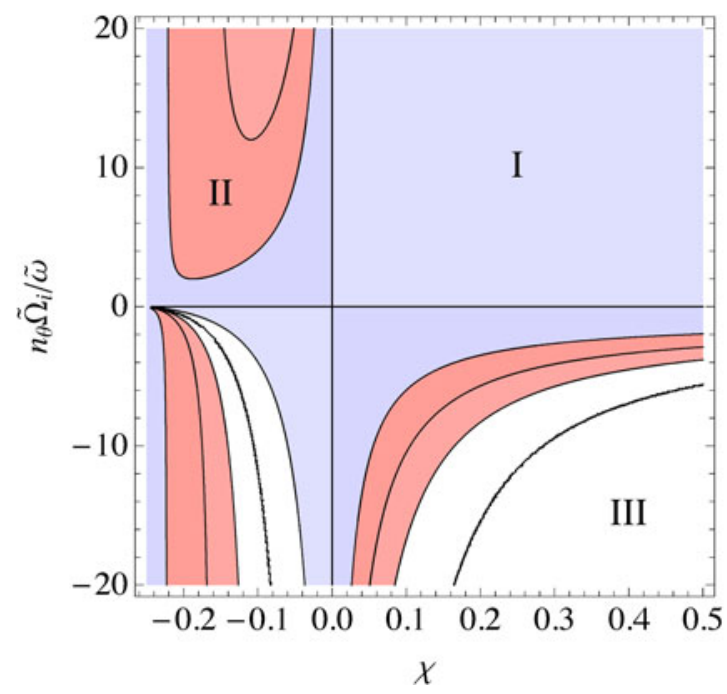

Fig. 1. Classification of wave interaction versus $\chi=\Omega_{E} / \Omega_{i}$ and $n_{\theta} \widetilde{\Omega}_{i} / \widetilde{\omega}$. In Region I, energy transfer is primarily between the kinetic and wave energy; in Region II, energy transfer is between kinetic and potential energy; and in Region III it is between wave and potential energy. The lines in the middle of the regions are $f_{E}=0$, $f_{E}=1$, and $\left|f_{E}\right| \rightarrow \infty$, respectively.

constant. If we consider a particle in the rest frame, we find that its energy, $E=W+q \Phi$, must be conserved since $v_{p h}=0$. The particle may scatter off the stationary wave in the $x-y$ plane, which allows stochastic motion in the $y$-direction.

\section{Region III}

In this case, $\left|f_{E}\right| \gg 1$, so the wave energy is primarily converted to potential energy. We see that $\left|f_{E}\right| \rightarrow$ $\infty$ if the wave is traveling with the same speed as the moving frame, $v_{p h}=v_{E}$. Thus, the wave does not change the particle's kinetic energy in the rotating frame $\widetilde{W}$ but breaks the azimuthal symmetry and provides a source of angular momentum, by which the particle can travel radially.

\section{PRODUCING ROTATION}

We have described the interaction of a single particle with a wave, but we have not yet detailed how to use these waves to produce rotation in a mirror. First, we will complete the picture of diffusion paths in a full mirror machine. Then, we will discuss how to fulfill two criteria necessary for alpha channeling. ${ }^{31}$ The diffusion paths must be favorable for driving rotation - there must be a population inversion along the diffusion path in the desired direction. Also, it is necessary to limit the energy a particle can gain from the wave to prevent losing significant rotation energy to few particles.

When particles interact with a specific wave, the perpendicular and parallel energy changes are related through $k_{\perp}$ and $k_{\|}$(Refs. 30 and 34). The potential energy change is related to the perpendicular energy change through the branching ratio. Knowing these changes in the wave region, it is easy to then determine the perpendicular, parallel, and potential energy change at the midplane. ${ }^{16}$ These will uniquely determine particle orbits if we ignore gyroangle, azimuthal angle, and bounce position. Repeatedly interacting with the wave at random phase thus produces diffusion along a one-dimensional path in $\widetilde{W}_{\perp 0^{-}}-\widetilde{W}_{10}-\Phi_{0}$ space.

A wave located at a specific position along the axis of a mirror has magnetic field $B_{0}<B<B_{m}$, where $B_{0}$ is the midplane magnetic field and $B_{m}$ is the mirror (maximum) magnetic field. Particles that are resonant with the wave satisfy the relation $\widetilde{\omega}-k_{\|} v_{\|}=\widetilde{\Omega}_{i}$ locally. In rotating midplane coordinates, the resonance is a line with slope $\left(R_{r f}-1\right)^{-1}$ where $R_{r f}=B / B_{0}$, that is, the same slope as the loss cone for that mirror ratio (Fig. 2). We can thus use waves placed at different mirror ratios to be resonant with particles in different regions of phase-space, as seen in Fig. 3 (Refs. 31 and 32).

Three diffusion paths are apparent in Fig. 2 that allow us to remove trapped particles through either the loss cone or the outer radius of the plasma. Path (a) to the loss cone reduces both the kinetic and potential energy of the particle, path (b) to the outer radius increases potential energy but decreases kinetic energy, and path (c) increases both kinetic and potential energy. In this diagram we have assumed that $\chi>0$ or $E_{r}<0$. This polarity has been somewhat more common in rotating mirrors. ${ }^{1} \chi>0$ is also preferred in this application since path (b), which

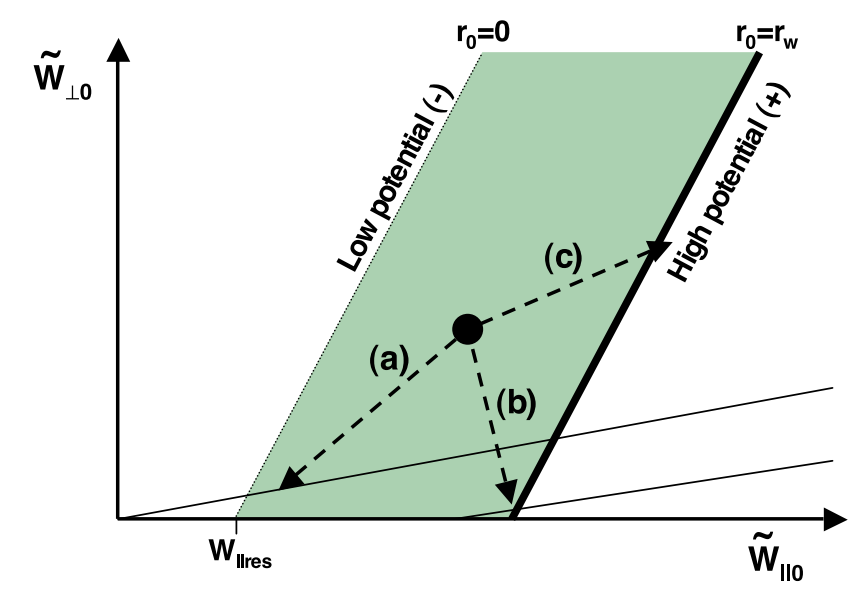

Fig. 2. The shaded region indicates the wave resonance, dependent on radius. The dashed lines depict diffusion paths that could eject particles from the plasma. 


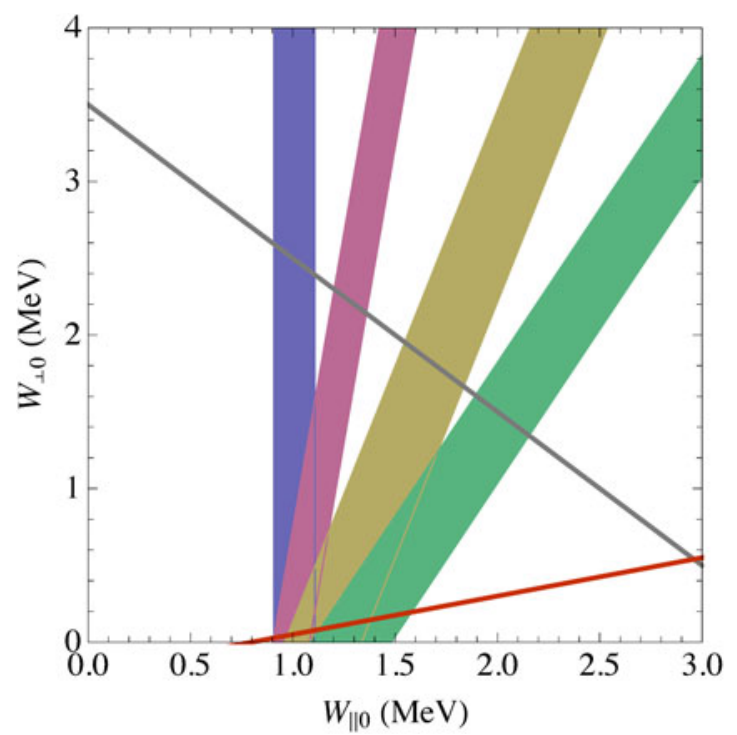

Fig. 3. Several wave regions are used to remove alpha particles across phase-space. The line at $3.5 \mathrm{MeV}$ indicates the alpha-particle birth energy, and the upward sloped line indicates the alpha particle loss cone. The properties of the waves are described in Table I.

produces rotation, has no opposite path leading to a loss region.

We wish to create a population inversion along path (b) to drive rotation. The wave's resonant parallel energy must be greater than the energy required to overcome the centrifugal potential for the particle to exit the loss cone. On the other hand, the wave resonant parallel energy must be less than the energy at which new particles are created for there to be any particles in resonance. For perpendicular waves, the particle will remain in resonance with the same wave, and these simple conditions on the resonant energy are sufficient to ensure favorable diffusion toward the exit. For nonperpendicular waves, the overall diffusion path must still intersect the particle source and loss region, but the resonance region of a single wave has no such restriction.

The final requirement for driving rotation is that the particle energy gain is restricted. The energy gain may be limited in three ways. The simplest way is by the limited radial extent of the plasma. Particles move inward as they gain energy, and once particles reach the center axis they cannot move in further. Although this is a strong restriction on energy gain, for most configurations this energy is much higher than that imposed by the second method. ${ }^{32}$

The second way to restrict the energy gain is to use nonoverlapping waves with $k_{\perp} \rho_{s} \sim 1$, where $\rho_{s}$ is the gyroradius of source particles. The diffusion coefficient for resonance with the first cyclotron harmonic is proportional to $J_{1}\left(k_{\perp} \rho\right)^{2}$, which will go to zero at $k_{\perp} \rho \approx$ $3.83,7.02, \ldots$ As the particle increases in perpendicular energy, $\rho$ increases, as does $k_{\perp}=n_{\theta} / r$. The diffusion coefficient will thus approach zero at some point as the particle gains energy from the wave.

Energy gain is further limited by the fixed resonance region if the wave is not perpendicular. As the particle changes its perpendicular energy, it also changes its parallel energy until it is out of resonance $\left(\widetilde{\omega}-k_{\|} v_{\|}=\widetilde{\Omega}_{i}\right.$ is not satisfied). If there is no wave overlapping on the high-energy side of the diffusion path, the particle will cease to gain energy from the waves.

\section{ALPHA CHANNELING}

In a fusion device, alpha particles provide a significant free energy source. The concept of alpha channeling is to use waves to extract alpha particles from the system collisionlessly at low energy. ${ }^{30}$ In a tokamak, the energy transferred to the wave can be used to drive electron currents or to heat fuel ions. ${ }^{35}$ Rapid removal of alpha particles from the system may double the fusion reactivity, and by redirecting their energy the heating efficiency is also improved. ${ }^{36}$ The concept has now been extended to mirror confinement devices. . $^{31,32}$

Supersonically rotating mirrors are especially interesting as fusion devices since they combine the benefits of axisymmetric mirrors with the possibility of high confinement. ${ }^{1-3}$ The benefits include simple construction, high beta, and naturally steady-state operation. By including supersonic rotation, one adds dramatically increased confinement, stability to MHD and loss-cone instabilities, and a natural heating mechanism. ${ }^{8-11} \mathrm{~A}$ primary reason centrifugal traps have been unable to perform as fusion devices has been the limitation due to the Alfven CIV (Refs. 1, 2, 37, and 38).

If we use alpha-particle energy to drive rotation, we may solve three significant problems simultaneously. ${ }^{16}$ First, the alpha particles would be removed rapidly, increasing the fusion reactivity as in stationary devices. Second, the efficiency will improve as rotation energy would not need to be provided externally. Finally, the end electrodes will not be needed, which is advantageous for technical reasons.

In order to drive rotation, we will need to use waves in Regions II or III of Fig. 1, and since $\chi>0$ we will be considering waves in the bottom right quadrant of that diagram. A simple way to produce these waves is to use a magnetic perturbation that is stationary in the lab frame, $\omega=0$. Stationary waves conserve energy in the rest frame, so $f_{E}=1$, and these waves will convert the kinetic energy of alpha particles into rotation energy.

Alpha particles are trapped by the mirror force in a reactor, so perpendicular waves are necessary to remove them at a low energy. The resonance condition then requires $\left|n_{\theta}\right| \approx \chi^{-1}$. As $\chi \sim 0.05$ for reasonable reactor parameters, this implies large wave numbers $n_{\theta} \sim 20$ 
TABLE I

Properties of the Stationary Waves Simulated for Alpha Channeling

\begin{tabular}{|l|c|c|c|c|}
\hline$R_{r f}$ & $n_{\theta}$ & $\begin{array}{c}k_{\|} \\
\left(\mathrm{cm}^{-1}\right)\end{array}$ & $\begin{array}{c}L \\
(\mathrm{~cm})\end{array}$ & $\begin{array}{c}B_{z}\left(r_{\max }\right) \\
(\mathrm{G})\end{array}$ \\
\hline 1.0 & -14 & -0.062 & 1000 & 60 \\
1.13 & -16 & -0.072 & 880 & 40 \\
1.3 & -19 & -0.074 & 420 & 10 \\
1.5 & -26 & -0.054 & 580 & 100 \\
\hline
\end{tabular}

(Ref. 3). In a vacuum, a perturbation of this type will decay like $r^{n_{\theta}}$. While this is a rapid decay, the plasma exists only near the cylinder boundary in a layer of thickness $a$. The ideal ratio $a / r \sim 0.1$ to 0.3 , so that the inside of the wave with $n_{\theta}=20$ will have decayed by a factor of 10 to 1000 assuming the vacuum decay. Clearly, this is a significant loss, but only a small magnetic perturbation $(<1 \mathrm{G})$ is necessary for alpha channeling to be faster than the collisional slowing-down time.

To demonstrate how these waves can be used for alpha channeling, we simulated alpha particles in an example reactor using the full equations of motion. The reactor has midplane magnetic field $B_{0}=2 \mathrm{~T}$, a mirror ratio of $5, \chi=0.05$, an inner radius of $125 \mathrm{~cm}$, and an outer radius of $150 \mathrm{~cm}$. This leads to a deuterium rotation energy of $500 \mathrm{keV}, T_{i}=100 \mathrm{keV}$ and $T_{e}=30 \mathrm{keV}$ according to the scaling in Ref. 3 . We assumed a plasma density of $1.4 \times 10^{13} \mathrm{~cm}^{-3}$ and estimated the plasma response using the cold plasma dispersion relation. ${ }^{39}$

For alpha channeling, we chose to use four waves to remove alpha particles from a large fraction of phasespace (see Table I and Fig. 3). The results of the simulation are shown in Fig. 4. On average, resonant alpha particles were removed at $1.3 \mathrm{MeV}$. The remaining 2.2 $\mathrm{MeV}$ per alpha particle was converted into potential energy.

These results confirm the theory and demonstrate that alpha-particle energy can be converted into rotation energy. The energy provided to the plasma by alpha particles must equal the energy loss rate. This implies that without alpha channeling, the reactor must operate at $\eta Q=6$, where $\eta$ is the fraction of alpha-particle energy retained in the plasma and $Q$ is the fusion power output over power input. This is not unreasonable for a centrifugal confinement scheme. ${ }^{3}$ With alpha channeling, in this case, no recirculating power is necessary $(Q=\infty)$.

\section{PLASMA CENTRIfUge}

Another application for supersonically rotating plasmas is isotope separation. Species of different mass have

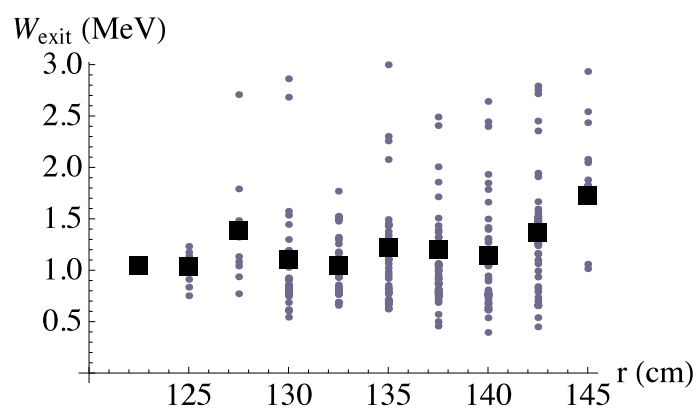

(a)

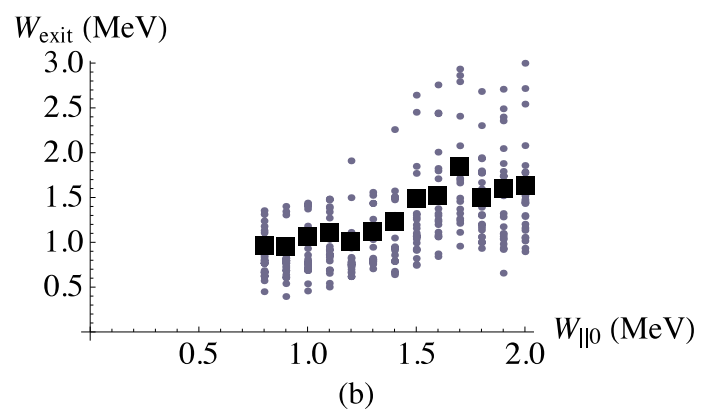

Fig. 4. The energy at which alpha particles are removed: (a) versus initial radius and (b) versus initial midplane parallel energy. Large squares indicate the mean, and dots represent single particles.

different rotation speeds in a rotating mirror trap due to the centrifugal drift, and drag between the species drives radial separation. ${ }^{4}$ Because higher rotation speeds may be obtained in a plasma than in a gas centrifuge, larger separation factors may be achieved. ${ }^{6,13,40}$

Early plasma centrifuges faced the same Alfven CIV limitation described earlier. ${ }^{5,12}$ Viscous heating leads to an additional limit on the separation factor that can be achieved in partially ionized plasmas. ${ }^{41}$ Because the product and waste must be collected along field lines, design of electrodes is further complicated by reactivity between the separated material and the electrode surface. ${ }^{42}$

The later development of the vacuum-arc plasma centrifuge solved many of these problems by using a pulsed, fully ionized plasma. ${ }^{6}$ The higher rotation velocities resulted in significantly higher separation factors than could be achieved in gas centrifuges. ${ }^{20}$ However, the devices have limited throughput due to their pulsed nature and small diameter. ${ }^{21,23}$ The separation factor could be further improved by using a convective (counterstreaming) flow pattern. . $^{17,22}$

We have suggested an electrodeless design for plasma centrifuges that relies on wave energy to provide rotation. ${ }^{17}$ By doing so, we expect to avoid the Alfven CIV limit along with other electrode issues, while retaining the advantages of a steady-state plasma centrifuge with counterstreaming flow. Driving rotation using wave energy requires waves in Region III of Fig. 1. Because 
isotopes separated with this method will be much heavier than hydrogen, values of $|\chi| \sim 1$ may be achieved for reasonable electric fields.

Unlike the alpha channeling case, it is not necessary to use perpendicular waves to remove particles at low energy through the loss cone. In order to produces waves with large $\left|f_{E}\right|$, we may thus use waves with small values of $\widetilde{\omega} / \widetilde{\Omega}_{i}$ rather than relying on large mode numbers $n_{\theta}$.

Since the collision time is on the order of several cyclotron times, waves with specific diffusion paths will not lead to a significantly non-Maxwellian distribution of particles. This by no means limits our ability to drive rotation. Particles will still diffuse along the same paths and will move preferentially down the density gradient in phase-space.

By injecting particles near the center of the trap, a radial density gradient may be created to drive rotation (Fig. 5). Particles will diffuse outward along the diffusion path until an equilibrium is reached between the rate at which the particle energy changes and the density gradient. If $n_{\theta}<0$, particles cool as they move outward and equilibrium will occur for Gaussian density profiles with $r=\rho_{i} \sqrt{-n_{\theta} \widetilde{\Omega}_{i} / \widetilde{\omega}}$. As particles that gain energy move inward, waves with $n_{\theta}<0$ also have a natural limit on the heating of ions. It is possible instead to heat ions as they move outward by using waves with $n_{\theta}>0$. In this case, there would be no limit on the radius or energy gain unless the wave could be localized radially.

Because the plasma radius is limited to $\rho_{i} \sqrt{-n_{\theta} \widetilde{\Omega}_{i} / \widetilde{\omega}}$, low frequency waves with $\widetilde{\omega} \ll \widetilde{\Omega}_{i}$ should be used to drive rotation. The waves must also have $\left|n_{\theta}\right| \geq 1$. It remains to identify a wave that satisfies these conditions and could be driven by an external antenna. In the past, it was found that the mode converted ion Bernstein wave would be effective at alpha channeling in tokamaks. ${ }^{43,44}$ In mirrors, one might instead use quasi-

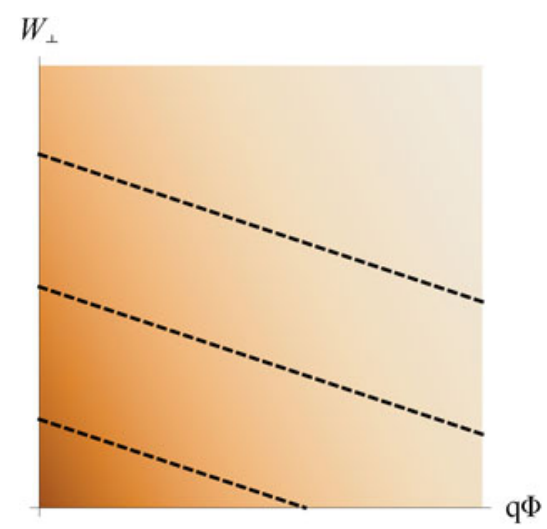

Fig. 5. Phase-space density plot with diffusion paths to drive rotation drawn (dashed lines). The $x$-axis variable is related to the radial coordinate by $q \Phi \propto r^{2}$. The temperature everywhere is constant. longitudinal or quasi-transverse kinetic Alfven waves. ${ }^{45}$ In the present case, an additional possibility is the resistive drift wave that has been observed in vacuum-arc plasma centrifuges. ${ }^{46}$

\section{CONCLUSIONS}

To summarize, we have shown that it is possible to maintain the angular momentum of supersonically rotating mirror machines using radio-frequency waves. In principle, the rotation energy may come from an arbitrary combination of kinetic energy and wave energy. Because electrodes are not necessary, it is possible that past limits on rotation speed will not affect devices driven this way.

Two specific applications of these waves were discussed. In a fusion reactor, waves were found that convert alpha-particle kinetic energy to rotation energy. This is an extension of the alpha channeling effect. Waves produced by a fixed magnetic ripple were shown to convert kinetic energy to potential energy as predicted. The practical details of this scheme, such as the spatial structure of the ripple and optimized values for a reactor, are the subject of ongoing research.

The second application considered was isotope separation. In this case, there is no kinetic energy source, so rotation must be created with wave energy. Eliminating electrodes in this case has the added benefit of reducing interaction with the outward flow of separated material. Waves meeting some criteria for driving rotation have been identified in experiments, but whether these waves will be practical or effective remains to be studied.

The achievement of supercritical rotation velocities in centrifugal mirror traps remains an exciting and so far elusive possibility. If the effects proposed here are successful in achieving these velocities, significant new opportunities will be opened in the areas of open trap magnetic confinement fusion and isotope separation, as well as other areas.

\section{ACKNOWLEDGMENT}

This work was supported by U.S. Department of Energy contracts DE-FG02-06ER54851 and DE-AC0276-CH03073.

\section{REFERENCES}

1. B. LEHNERT, "Rotating Plasmas," Nucl. Fusion, 11, 485 (1971).

2. B. LEHNERT, "On the Possibilities of Plasmas Rotating at Super-Critical Velocities,” Phys. Scr., 9, 189 (1974).

3. A. A. BEKHTENEV et al., "Problems of a Thermonuclear Reactor with a Rotating Plasma," Nucl. Fusion, 20, 579 (1980). 
4. B. BONNEVIER, "Diffusion due to Ion-Ion Collisions in a Multicomponent Plasma," Ark. Fys., 33, 255 (1967).

5. B. BONNEVIER, "Experimental Evidence of Element and Isotope Separation in a Rotating Plasma," Plasma Phys., 13, 763 (1971).

6. M. KRISHNAN et al., "Plasma Centrifuge," Phys. Rev. Lett., 46, 36 (1981).

7. T. OHKAWA and R. MILLER, "Band Gap Ion Mass Filter," Phys. Plasmas, 9, 5116 (2002).

8. V. N. BOCHAROV et al., "MHD-Stable Confinement of a Rotating Plasma,” JETP Lett., 41, 601 (1985).

9. V. P. PASTUKHOV, Reviews of Plasma Physics, Vol. 13, pp. 203-259, Consultants Bureau, New York (1987).

10. D. D. RYUTOV, "Open Ended Traps," Sov. Phys. Usp., 31, 300 (1988).

11. R. F. ELLIS et al., "Steady Supersonically Rotating Plasmas in the Maryland Centrifugal Experiment," Phys. Plasmas, 12, 55704 (2005).

12. N. NATHRATH et al., "Isotope Separation in Rotating Plasmas," Uranium Isotope Separation: Proc. Int. Conf., 53 (1975).

13. M. W. GROSSMAN and T. A. SHEPP, "Plasma IsotopeSeparation Methods," IEEE Trans. Plasma Sci., 19, 1114 (1991).

14. H. ALFVÉN, "Collision Between a Nonionized Gas and a Magnetized Plasma," Rev. Mod. Phys., 32, 710 (1960).

15. N. BRENNING, "Review of the CIV Phenomenon," Space Sci. Rev., 59, 209 (1992).

16. A. J. FETTERMAN and N. J. FISCH, "Alpha Channeling in a Rotating Plasma," Phys. Rev. Lett., 101, 205003 (2008).

17. A. J. FETTERMAN and N. J. FISCH, "Wave-Driven Countercurrent Plasma Centrifuge," Plasma Sources Sci. Technol., 18, 045003 (2009).

18. S. T. LAI, "A Review of Critical Ionization Velocity," Rev. Geophys., 39, 471 (2001).

19. G. F. ABDRASHITOV et al., "Hot Rotating Plasma in the PSP-2 Experiment,” Nucl. Fusion, 31, 1275 (1991).

20. E. D. BOSCO et al., "Isotopic Enrichment in a Plasma Centrifuge," Appl. Phys. Lett., 50, 1716 (1987).

21. M. KRISHNAN and R. PRASAD, "Parametric Analysis of Isotope Enrichment in a Vacuum-Arc Centrifuge," J. Appl. Phys., 57, 4973 (1985).

FUSION SCIENCE AND TECHNOLOGY VOL. $57 \quad$ MAY 2010
22. A. A. IVANOV and N. N. TIMCHENKO, "Counterstreaming Mass Separation of Ions in a Fully Ionized Rotating Plasma," Soviet J. Plasma Phys., 16, 863 (1990).

23. M. HOLE and S. W. SIMPSON, "Separative Performance in a Vacuum Arc Centrifuge," IEEE Trans. Plasma Sci., 27, 620 (1999).

24. A. A. BEKHTENEV and V. I. VOLOSOV, "Generation of a Radial Electric Field in a Rotating Plasma," Soviet Phys. Tech. Phys., 23, 938 (1978).

25. K. IDA, "Experimental Studies of the Physical Mechanism Determining the Radial Electric Field and Its Radial Structure in a Toroidal Plasma," Plasma Phys. Control. Fusion, 40, 1429 (1998).

26. C. S. CHANG et al., "Generation of Plasma Rotation by Ion Cyclotron Resonance Heating in Tokamaks," Phys. Plasmas, 6, 1969 (1999).

27. F. W. PERKINS et al., "Generation of Plasma Rotation in a Tokamak by Ion-Cyclotron Absorption of Fast Alfven Waves," Phys. Plasmas, 8, 2181 (2001).

28. L. G. ERIKSSON et al., "Plasma Rotation Induced by Directed Waves in the Ion-Cyclotron Range of Frequencies," Phys. Rev. Lett., 92, 235001 (2004).

29. S. I. BRAGINSKII, "Transport Processes in a Plasma," Rev. Plasma Phys., 1, 205 (1965).

30. N. J. FISCH and J. M. RAX, "Interaction of Energetic Alpha Particles with Intense Lower Hybrid Waves," Phys. Rev. Lett., 69, 612 (1992).

31. N. J. FISCH, "Alpha Channeling in Mirror Machines," Phys. Rev. Lett., 97, 225001 (2006).

32. A. I. ZHMOGINOV and N. J. FISCH, "Simulation of $\alpha$-Channeling in Mirror Machines," Phys. Plasmas, 15, 042506 (2008).

33. B. LEHNERT, Dynamics of Charged Particles, Interscience Publishers, New York (1964).

34. L. CHEN et al., "Ion Radial Transport Induced by ICRF Waves in Tokamaks," Nucl. Fusion, 28, 389 (1988).

35. M. C. HERRMANN and N. J. FISCH, "Cooling Energetic Alpha Particles in a Tokamak with Waves," Phys. Rev. Lett., 79, 1495 (1997).

36. N. J. FISCH and M. C. HERRMANN, "Utility of Extracting Alpha Particle Energy by Waves," Nucl. Fusion, 34, 1541 (1994)

37. B. LEHNERT, "On the Equilibrium and Stability of Rotating High-Beta Plasmas,” Phys. Scr., 9, 229 (1974).

38. C. TEODORESCU et al., "Experimental Study on the Velocity Limits of Magnetized Rotating Plasmas," Phys. Plasmas, 15, 042504 (2008). 
39. T. H. STIX, Waves in Plasmas, American Institute of Physics, New York (1992).

40. M. BENEDICT, Nuclear Chemical Engineering, McGraw Hill, New York (1981).

41. M. M. B. WIJNAKKER and E. H. A. GRANNEMAN, "Limitations on Mass Separation by the Weakly Ionized Plasma Centrifuge," Z. Naturforsch, 35a, 883 (1980).

42. E. A. WITALIS, "Electrodeless Plasma Isotope Centrifuge," Atomkernenerg. Kerntech., 38, 32 (1981).
43. E. J. VALEO and N. J. FISCH, "Excitation of Large- $k \theta$ Ion-Bernstein Waves in Tokamaks," Phys. Rev. Lett., 73, 3536 (1994).

44. N. J. FISCH, "Alpha Power Channeling Using Ion Bernstein Waves," Phys. Plasmas, 2, 2375 (1995).

45. A. I. ZHMOGINOV and N. J. FISCH, "Waves for AlphaChanneling in Mirror Machines," Phys. Plasmas, 16, 112511 (2009).

46. M. J. HOLE et al., "Plasma Instability of a Vacuum Arc Centrifuge,” Phys. Rev. E, 65, 933 (2002). 